\title{
Highly Convergent Route to Cyclopeptide Alkaloids. Total Synthesis of Ziziphine N
}

\author{
Gang He, Jing Wang and Dawei Ma*
}

State Key Laboratory of Bioorganic and Natural Products Chemistry, Shanghai

Institute of Organic Chemistry, Chinese Academy of Sciences, 354 Fenglin Lu, Shanghai 200032, China

Supporting Information

Table of contents

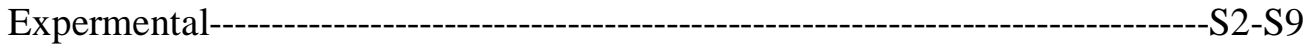

Copies of NMR spectrum of compounds $\mathbf{1 , 2}, \mathbf{4}$, and 10-12------------------S10-S17 


\section{Experimental}

Synthesis of 7. To a solution of 6 (5.0 g, $15.7 \mathrm{mmol})$, Meldrum's acid (2.25 g, $15.7 \mathrm{mmol})$ and DMAP (2.87 $\mathrm{g}, 23.5 \mathrm{mmol})$ in $80 \mathrm{~mL}$ of methylene chloride was added DCC (3.9 g, $18.8 \mathrm{mmol})$ at $0{ }^{\circ} \mathrm{C}$. The temperature was slowly raised to room temperature and stirring was continued for $3 \mathrm{~h}$. The mixture was filtered and the filtration was diluted with $800 \mathrm{~mL}$ cold ethyl acetate. The solution was washed subsequently with cold $5 \% \mathrm{KHSO}_{4}$ solution, water and brine, dried over anhydrous

$\mathrm{Na}_{2} \mathrm{SO}_{4}$. The solvent was evaporated and the residue was refluxed with $200 \mathrm{~mL}$ of methanol for 40 min. The solvent was evaporated again and the residue was dissolved in $80 \mathrm{~mL}$ dichloromethane, sodium borohydride $(1.0 \mathrm{~g}, 26.6 \mathrm{mmol})$ was added portion-wise at $0{ }^{\circ} \mathrm{C}$. Stirring was continued at $0{ }^{\circ} \mathrm{C}$ for $8 \mathrm{~h}$, the reaction was quenched with $100 \mathrm{~mL}$ water. After common work-up and flash chromatography, $2.8 \mathrm{~g} \mathrm{(50 \% )} \mathrm{of}$ 7 was obtained. . ${ }^{1} \mathrm{H}$ NMR $\left(300 \mathrm{MHz}, \mathrm{CDCl}_{3}\right) \delta 0.06(\mathrm{~d}, J=6.3 \mathrm{~Hz}, 6 \mathrm{H}), 0.89(\mathrm{~s}, 9 \mathrm{H})$, $1.50(\mathrm{~s}, 9 \mathrm{H}), 2.57(\mathrm{dd}, J=8.1,15.4 \mathrm{~Hz}, 1 \mathrm{H}), 2.79(\mathrm{dd}, J=9.3 \mathrm{~Hz}, 18 \mathrm{~Hz}, 1 \mathrm{H}), 2.88(\mathrm{bs}$, $1 \mathrm{H}), 4.10(\mathrm{~d}, J=2.7 \mathrm{~Hz}, 2 \mathrm{H}), 4.18(\mathrm{dt}, J=7.8 \mathrm{~Hz}, 2.7 \mathrm{~Hz}, 1 \mathrm{H}), 4.55-4.58(\mathrm{~m}, 1 \mathrm{H})$; ESI-MS $m / z 368.4[\mathrm{M}+\mathrm{Na}]^{+}$.

Reduction of 7. To a solution of $7(415 \mathrm{mg}, 1.2 \mathrm{mmol})$ in $6 \mathrm{~mL}$ anhydrous THF at room temperature was added boran-dimethylsulfide $(0.36 \mathrm{~mL}, 3.6 \mathrm{mmol})$. The mixture was refluxed for $4 \mathrm{~h}$. The reaction mixture was cooled to room temperature, diluted with ether $(50 \mathrm{~mL})$, and quenched with saturated ammonium chloride $(5 \mathrm{~mL})$. The organic phase was separated and washed with $0.1 \mathrm{~N} \mathrm{HCl}$, saturated $\mathrm{NaHCO}_{3}$ and brine, dried over $\mathrm{Na}_{2} \mathrm{SO}_{4}$, and evaporated to dryness. The residue was purified by 
column chromatography to give 3 (376 mg, $90 \%$ yield). ${ }^{1} \mathrm{H}$ NMR $\left(300 \mathrm{MHz}, \mathrm{CDCl}_{3}\right) \delta$ $008(\mathrm{~d}, J=3.9 \mathrm{~Hz}, 6 \mathrm{H}), 0.89(\mathrm{~s}, 9 \mathrm{H}), 1.46(\mathrm{~s}, 9 \mathrm{H}), 1.91-1.97(\mathrm{~m}, 1 \mathrm{H}), 2.02-2.08(\mathrm{~m}$, 1H), 3.40-3.45 (m, 3H), 3.77-4.10 (m, 3H), 4.44-4.49 (m, 1H).

Preparation of aldehyde 9. The solution of $8(6.0 \mathrm{~g}, 30.9 \mathrm{mmol})$ and sodium hydroxide $(9.9 \mathrm{~g}, 247 \mathrm{mmol})$ in $100 \mathrm{~mL}$ ethanol/ $\mathrm{H}_{2} \mathrm{O}(2: 1)$ was heated to $70{ }^{\circ} \mathrm{C}$, chloroform $(5.0 \mathrm{~mL}, 61.8 \mathrm{mmol})$ was added over $1 \mathrm{~h}$. The resulting mixture was stirred $3 \mathrm{~h}$, cooled to room temperature, evaporated to remove ethanol. The left water solution was adjust to $\mathrm{pH} 4 \sim 5$, extracted with ethyl acetate. Common work-up and purification gave $2.1 \mathrm{~g}$ (41\% yield) of aldehyde, which was dissolved in $10 \mathrm{~mL}$ DMF. To this solution $\mathrm{K}_{2} \mathrm{CO}_{3}(1.6 \mathrm{~g}, 11.3 \mathrm{mmol})$ and methyl iodide $(0.7 \mathrm{~mL}, 11.3 \mathrm{mmol})$ were added. The mixture was then stirred at room temperature for $1 \mathrm{~h}$, quenched with water. Common work-up and purification gave product 9 (1.9 g, 87\% yield). ${ }^{1} \mathrm{H}$ NMR (300 $\left.\mathrm{MHz}, \mathrm{CDCl}_{3}\right) \delta 1.68-1.87(\mathrm{~m}, 7 \mathrm{H}), 2.82-2.85(\mathrm{~m}, 1 \mathrm{H}), 3.90(\mathrm{~s}, 3 \mathrm{H}), 5.35(\mathrm{t}, J=3.0 \mathrm{~Hz}$ $1 \mathrm{H}), 6.93(\mathrm{~d}, J=9.0 \mathrm{~Hz}, 1 \mathrm{H}), 7.27(\mathrm{dd}, J=9.0,3.0 \mathrm{~Hz}, 1 \mathrm{H}), 7.51(\mathrm{~d}, J=3.3 \mathrm{~Hz}, 1 \mathrm{H})$, $10.90(\mathrm{~s}, 1 \mathrm{H}) ;{ }^{13} \mathrm{C} \mathrm{NMR}\left(75 \mathrm{MHz}, \mathrm{CDCl}_{3}\right) \delta 18.82,25.15,30.30,56.01,62.11,97.15$ $112.88,115.38,117.52,124.96,125.11,150.87,157.09,189.38 ; \mathrm{MS} m / z 259.1[\mathrm{M}+$ $\mathrm{Na}]^{+}$; HRMS Calcd. for $\mathrm{C}_{13} \mathrm{H}_{16} \mathrm{O}_{4} \mathrm{Na}[\mathrm{M}+\mathrm{Na}]^{+}$259.0948, found 259.0941. IR (KBr) $1027,1162,1220,1282,1496,1676,2879,2941 \mathrm{~cm}^{-1}$.

Synthesis of vinyl iodide 4. To a suspension of iodomethyltriphenylphosphonium iodide $(10.7 \mathrm{~g}, 20.3 \mathrm{mmol})$ in $60 \mathrm{~mL}$ anhydrous THF at room temperature was slowly added $20.3 \mathrm{~mL}$ of a $1 \mathrm{M}$ solution of lithium hexamethyldisilazane in THF. After stirring for $10 \mathrm{~min}$, the solution was cooled to -78 
${ }^{\circ} \mathrm{C}$ and HMPA (5.0 mL) was added. It was stirred for another $10 \mathrm{~min}$ before a solution of aldehyde 9 (4.0 g, $16.9 \mathrm{mmol})$ in $15 \mathrm{~mL}$ THF was slowly added. After addition was completed, the mixture was stirred at the same temperature for $10 \mathrm{~min}$, then warmed to room temperature, and stirred for another $1 \mathrm{~h}$. The mixture was diluted with $500 \mathrm{~mL}$ of hexane and then filtered. The filtrate was washed with brine, dried over $\mathrm{Na}_{2} \mathrm{SO}_{4}$ and evaporated to dryness. The residue was dissolved in $50 \mathrm{~mL}$ methanol before PPTS (200 mg) was added. The reaction was monitored until the substrate was completely consumed. Common work-up and purification give product 4 (3.3 g, 56\% yield). ${ }^{1} \mathrm{H}$ NMR (300 MHz, $\left.\mathrm{CDCl}_{3}\right) \delta 3.78(\mathrm{~s}, 3 \mathrm{H}), 4.87$ (bs, 1H), $6.58(\mathrm{dd}, J=2.1,8.4 \mathrm{~Hz}, 1 \mathrm{H})$, 6.75-6.84 (m, 2H), 7.26-7.38 (m, 2H); ${ }^{13} \mathrm{C}$ NMR (75 MHz, $\left.\mathrm{CDCl}_{3}\right) \delta$ 56.28, 81.24, 112.13, 115.72, 116.05, 126.87, 134.46, 148.63, 151.21; MS m/z $277.0[\mathrm{M}]^{+}$, HRMS Calcd. for $\mathrm{C}_{9} \mathrm{H}_{10} \mathrm{O}_{2} \mathrm{I}$ 276.9718, found 276.9720; IR (KBr) 1031, 1217, 1491, 1600, $2835,2939,3348 \mathrm{~cm}^{-1}$

Mitsunobu reaction of 3 and 4. DIAD $(0.75 \mathrm{~mL}, 3.6 \mathrm{mmol})$ was added dropwise to a solution of $3(600 \mathrm{mg}, 1.8 \mathrm{mmol}), 4(600 \mathrm{mg}, 2.2 \mathrm{mmol})$, and triphenylphosphine $(950 \mathrm{mg}, 3.6 \mathrm{mmol})$ in THF $(30 \mathrm{~mL})$. The mixture was heated at $80{ }^{\circ} \mathrm{C}$ for $3 \mathrm{~h}$ and the solvent was evaporated. The crude product was extracted with ether $(25 \mathrm{~mL})$ and filtered. The solution was dried and concentrated under reduced pressure. Chromatography on silica gel gave 10 (570 mg, 54\% yield). ${ }^{1} \mathrm{H}$ NMR (300 $\left.\mathrm{MHz}, \mathrm{CDCl}_{3}\right) \delta 0.71(\mathrm{~s}, 6 \mathrm{H}), 0.91(\mathrm{~s}, 9 \mathrm{H}), 1.47(\mathrm{~s}, 9 \mathrm{H}), 2.15-2.19(\mathrm{~m}, 2 \mathrm{H}), 3.46-3.59$ (m, 2H), $3.79(\mathrm{~s}, 3 \mathrm{H}), 3.85-3.89(\mathrm{~m}, 2 \mathrm{H}), 3.94-4.05(\mathrm{~m}, 2 \mathrm{H}), 4.81(\mathrm{~d}, J=9.3 \mathrm{~Hz}, 1 \mathrm{H})$, $6.58(\mathrm{~d}, J=8.4 \mathrm{~Hz}, 1 \mathrm{H}), 6.78(\mathrm{~d}, J=7.8 \mathrm{~Hz}, 1 \mathrm{H}), 9.32(\mathrm{~m}, 1 \mathrm{H}), 7.33-7.39(\mathrm{~m}, 2 \mathrm{H}) ;{ }^{13} \mathrm{C}$ 
NMR (75 MHz, $\left.\mathrm{CDCl}_{3}\right) \delta$-5.39, 14.09, 18.24, 21.76, 22.67, 25.84, 28.54, 30.12, 44.99, $56.07,62.35,62.94,64.27,80.41,111.78,116.33,117.15,134.73,150.45,151.46$, 154.55; MS m/z 612.1 $[\mathrm{M}+\mathrm{Na}]^{+}$; HRMS Calcd. for $\mathrm{C}_{25} \mathrm{H}_{40} \mathrm{NO}_{5} \mathrm{SiINa}[\mathrm{M}+\mathrm{Na}]^{+}$ 612.1613, found 612.1616; IR (KBr) 777, 836, 1116, 1227, 1393, 1508, 1698, 2857, 2930, $2955 \mathrm{~cm}^{-1} ;[\alpha]_{\mathrm{D}}^{22}=-12.7\left(c=0.84\right.$ in $\left.\mathrm{CHCl}_{3}\right)$.

Cross coupling of $\mathbf{1 0}$ and 5. A mixture of $\mathbf{1 0}(36 \mathrm{mg}, 0.06 \mathrm{mmol})$, $N$-alloxycarbonyl-L-proline amide 5 (18 mg, $0.09 \mathrm{mmol}), \mathrm{CuI}(12 \mathrm{mg}, 0.06 \mathrm{mmol})$, $\mathrm{N}, \mathrm{N}$-dimethylglycine hydrochloride $(9 \mathrm{mg}, 0.06 \mathrm{mmol})$, cesium carbonate ( $60 \mathrm{mg}, 0.18$ mmol) in dry 1,4-dioxane $(5 \mathrm{~mL})$ was heated to $80{ }^{\circ} \mathrm{C}$ under argon atmosphere for $12 \mathrm{~h}$. The cooled mixture was diluted with ethyl acetate $(50 \mathrm{~mL})$, filtered through celite. The filtrate was concentrated, and the residue was purified via chromatography to afford $\mathbf{1 1}$ (24 mg, 75\% yield). ${ }^{1} \mathrm{H}$ NMR (300 MHz, $\left.\mathrm{CDCl}_{3}\right) \delta 0.08(\mathrm{~s}, 6 \mathrm{H}), 0.92(\mathrm{~s}, 9 \mathrm{H}), 1.46(\mathrm{~s}$, 9H), 1.91 (s, 2H), 2.05-2.19 (m, 4H), 3.49 (m, 5H), $3.81(\mathrm{~s}, 3 \mathrm{H}), 3.87$ (m, 1H), 4.37 (s, 1H), $4.54(\mathrm{~s}, 2 \mathrm{H}), 4.80(\mathrm{~d}, J=8.7 \mathrm{~Hz}, 1 \mathrm{H}), 5.18(\mathrm{~m}, 2 \mathrm{H}), 5.65(\mathrm{~d}, J=10.2 \mathrm{~Hz}, 1 \mathrm{H})$, $5.88(\mathrm{~m}, 1 \mathrm{H}), 6.73(\mathrm{~s}, 1 \mathrm{H}), 6.88(\mathrm{~m}, 3 \mathrm{H}), 9.33(\mathrm{~d}, J=14.7 \mathrm{~Hz}, 1 \mathrm{H}) ;{ }^{13} \mathrm{C} \mathrm{NMR}(75 \mathrm{MHz}$, $\left.\mathrm{CDCl}_{3}\right) \delta-5.37,0.99,18.25,21.93,23.77,25.75,28.52,28.81,44.94,45.28,46.91$, $47.26,57.55,61.13,62.38,62.97,63.98,66.21,79.32,79.68,114.56,117.67,118.17$ 122.30, 126.18, 151.94, 154.53, 169.74; MS m/z 682.3 [M + Na $]^{+}$; HRMS Calcd. for $\mathrm{C}_{34} \mathrm{H}_{53} \mathrm{~N}_{3} \mathrm{O}_{8} \mathrm{SiNa}[\mathrm{M}+\mathrm{Na}]^{+}$682.3494, found 682.3496; IR (KBr) 775, 837, 1117, $1178,1398,1494,1651,1698,2857,2887,2954 \mathrm{~cm}^{-1} ;[\alpha]_{\mathrm{D}}{ }^{22}=-36.8(c=0.96$ in $\left.\mathrm{CHCl}_{3}\right)$.

Amino acid 12. To a solution of $11(140 \mathrm{mg}, 0.21 \mathrm{mmol})$ in THF (2 mL) was 
added $0.25 \mathrm{~mL}$ of $1.0 \mathrm{M}$ TBAF in THF. The resulting solution was stirred at room temperature until the substrate was completely consumed monitored by TLC. The reaction mixture was diluted with ethyl acetate $(20 \mathrm{~mL})$, washed with water and brine, and dried over $\mathrm{Na}_{2} \mathrm{SO}_{4}$. Common purification afford the corresponding alcohol (110 mg, 95\%). ${ }^{1} \mathrm{H}$ NMR (300 MHz, $\left.\mathrm{CDCl}_{3}\right) \delta 1.26(\mathrm{~s}, 9 \mathrm{H}), 1.91(\mathrm{~m}, 2 \mathrm{H}), 2.14-2.30(\mathrm{~m}$, $4 \mathrm{H}), 3.50-3.54(\mathrm{~m}, 5 \mathrm{H}), 3.75(\mathrm{~m}, 1 \mathrm{H}), 3.81(\mathrm{~s}, 3 \mathrm{H}), 4.37(\mathrm{~m}, 1 \mathrm{H}), 4.55(\mathrm{~m}, 2 \mathrm{H})$, 5.07-5.24 (m, 2H), $5.18(\mathrm{~m}, 2 \mathrm{H}), 5.67(\mathrm{~d}, J=9.9 \mathrm{~Hz}, 1 \mathrm{H}), 5.88(\mathrm{~m}, 1 \mathrm{H}), 6.75-6.88(\mathrm{~m}$, 4H), $8.94(\mathrm{~m}, 1 \mathrm{H}) ;{ }^{13} \mathrm{C} \mathrm{NMR}\left(75 \mathrm{MHz}, \mathrm{CDCl}_{3}\right) \delta 14.07,23.72,24.49,28.44,44.94$, $45.31,46.96,47.57,57.03,57.37,60.87,61.20,62.69,64.21,65.01,66.37,78.94$ $80.18,106.47,107.54,117.54,122.19,125.55,125.84,132.45,150.83,151.54,156.08$ $169.56 \mathrm{~cm}^{-1}$; MS m/z $568.2[\mathrm{M}+\mathrm{Na}]^{+}$; HRMS Calcd. for $\mathrm{C}_{28} \mathrm{H}_{39} \mathrm{~N}_{3} \mathrm{O}_{8} \mathrm{Na}[\mathrm{M}+\mathrm{Na}]^{+}$ 568.2629, found 568.2624; IR (KBr) 733, 772, 1122, 1222, 1405, 1495, 1693, 2888, 2976, 3420; $[\alpha]_{\mathrm{D}}^{22}=-33.7\left(c=0.97\right.$ in $\left.\mathrm{CHCl}_{3}\right)$.

A mixture of the above alcohol (110 $\mathrm{mg}, 0.2 \mathrm{mmol})$ and Dess-Martin periodinane $(128 \mathrm{mg}, 0.3 \mathrm{mmol})$ in dichloromethane $(10 \mathrm{~mL})$ was stirred at room temperature for $2 \mathrm{~h}$, quenched with saturated $\mathrm{Na}_{2} \mathrm{~S}_{2} \mathrm{O}_{3}$. The organic phase was separated and the aqueous phase was extracted with dichloromethane $(20 \mathrm{~mL} \times 3)$. The combined organic layers were washed with saturated $\mathrm{NaHCO}_{3}$ and brine, dried over $\mathrm{Na}_{2} \mathrm{SO}_{4}$, and evaporated to dryness. The residue was dissolved in the mixture of $t$-BuOH $(4 \mathrm{~mL})$, acetonitrile $(2 \mathrm{~mL})$ and 2-methyl-1-butene $(1 \mathrm{~mL})$. The mixture was cooled to $0{ }^{\circ} \mathrm{C}$ followed by addition of a newly prepared solution of $\mathrm{NaH}_{2} \mathrm{PO}_{4}(120 \mathrm{mg})$ and $\mathrm{NaClO}_{2}(100 \mathrm{mg})$ in water $(6 \mathrm{~mL})$. The mixture was then slowly warmed to room 
temperature before it was quenched with saturated $\mathrm{Na}_{2} \mathrm{~S}_{2} \mathrm{O}_{3}$. After common work-up, the product obtained was dissolved in dry methylene chloride $(10 \mathrm{~mL})$,. To this solution $\mathrm{Pd}\left(\mathrm{PPh}_{3}\right)_{4}(30 \mathrm{mg}, 0.02 \mathrm{mmol})$ and diethylamine $(100 \mu \mathrm{L}, 1 \mathrm{mmol})$ were added. After the mixture was stirred at room temperature for $50 \mathrm{~min}$, the solvent was removed and the residue was purified via silica gel column to afford amino acid 2 (56 mg, 56\% yield); ${ }^{1} \mathrm{H}$ NMR (300 MHz, $\left.\mathrm{CD}_{3} \mathrm{OD}\right) \delta 1.43(2 \mathrm{~s}, 9 \mathrm{H}), 2.08(\mathrm{~m}, 5 \mathrm{H}), 2.43(\mathrm{~m}$, 1H), $3.37(\mathrm{~m}, 2 \mathrm{H}), 3.55-3.75(\mathrm{~m}, 3 \mathrm{H}), 3.82(\mathrm{~s}, 3 \mathrm{H}), 4.44-4.47(\mathrm{~m}, 1 \mathrm{H}), 4.84(\mathrm{~m}, 1 \mathrm{H})$, $6.05(\mathrm{~d}, J=9.6 \mathrm{~Hz}, 1 \mathrm{H}), 6.77-6.97(\mathrm{~m}, 3 \mathrm{H}), 7.45(\mathrm{~d}, J=2.7 \mathrm{~Hz}, 1 \mathrm{H}) ;{ }^{13} \mathrm{C}$ NMR $(75$ $\left.\mathrm{MHz}, \mathrm{CD}_{3} \mathrm{OD}\right) \delta 25.45,29.05,30.71,31.43,32.23,46.20,46.58,47.46,57.06,61.91$, $69.41,81.34,83.96,84.49,109.95,110.61,114.13,116.92,117.49,119.25,122.00$ 125.94, 152.94, 153.46, $156.91 \mathrm{~cm}^{-1}$; MS m/z 476.1 $[\mathrm{M}+\mathrm{H}]^{+}$; HRMS Calcd. for $\mathrm{C}_{24} \mathrm{H}_{34} \mathrm{~N}_{3} \mathrm{O}_{7}[\mathrm{M}+\mathrm{H}]^{+}$476.2391, found 476.2381; IR (KBr) 776, 1170, 1220, 1417, $1614,1682,2928,2971 ;[\alpha]_{\mathrm{D}}^{22}=+29.1\left(c=0.83\right.$ in $\left.\mathrm{CH}_{3} \mathrm{OH}\right)$

Lactam 2. To a solution of FDPP (109 mg, $0.28 \mathrm{mmol})$, diisopropylethylamine (100 $\mu \mathrm{L}, 0.56 \mathrm{mmol})$ in $40 \mathrm{~mL}$ anhydrous DMF was added a solution of 12 (27 mg, $0.05 \mathrm{mmol})$ in dry DMF $(110 \mathrm{~mL})$ in a dropwise manner under Ar atmosphere. The mixture was stirred at room temperature for $24 \mathrm{~h}$ before the solvent was removed under reduced pressure. The residue was dissolved in ethyl acetate, washed with water and brine, and dried over $\mathrm{Na}_{2} \mathrm{SO}_{4}$. The solution was concentrated and the residue was chromatographed to give $2(17 \mathrm{mg}, 66 \%) ;{ }^{1} \mathrm{H}$ NMR $\left(300 \mathrm{MHz}, \mathrm{CDCl}_{3}\right) \delta 1.44(\mathrm{~s}, 9 \mathrm{H})$, 1.74-1.84 (m, 2H), 1.97-2.04 (m, 2H), 2.16-2.25 (m, 2H), $2.35(\mathrm{~m}, 1 \mathrm{H}), 3.31(\mathrm{~m}, 1 \mathrm{H})$ 3.39-3.47 (m, 1H), $3.80(\mathrm{~s}, 3 \mathrm{H}), 4.30(\mathrm{~m}, 2 \mathrm{H}), 4.63(\mathrm{~d}, J=5.7 \mathrm{~Hz}, 1 \mathrm{H}), 5.30(\mathrm{~m}, 1 \mathrm{H})$, 
$5.94(\mathrm{~d}, J=8.4 \mathrm{~Hz}, 1 \mathrm{H}), 6.81-6.96(\mathrm{~m}, 4 \mathrm{H}), 8.38(\mathrm{~d}, J=11.1 \mathrm{~Hz}, 1 \mathrm{H}) ;{ }^{13} \mathrm{C}$ NMR $(75$ $\left.\mathrm{MHz}, \mathrm{CDCl}_{3}\right) \delta 24.93,28.20,29.33,32.47,45.33,47.85,53.39,56.02,61.83,62.47$, $79.11,80.26,106.57,110.86,113.92,117.08,121.63,124.21,151.13,153.94,167.87$ 172.53; MS m/z 480.1 $[\mathrm{M}+\mathrm{Na}]^{+}$; HRMS Calcd. for $\mathrm{C}_{24} \mathrm{H}_{31} \mathrm{~N}_{3} \mathrm{O}_{6} \mathrm{Na}[\mathrm{M}+\mathrm{Na}]^{+}$ 480.2105, found 480.2098; IR (KBr) 731, 1053, 1131, 1224, 1398, 1509, 1640, 1655, $1696,2930,2976,3392 \mathrm{~cm}^{-1} ;[\alpha]_{\mathrm{D}}^{22}=-406.4\left(c=0.56\right.$ in $\left.\mathrm{CHCl}_{3}\right)$.

Ziziphine N 1. To a solution of $\mathbf{2}(25 \mathrm{mg}, 0.05 \mathrm{mmol})$ in dry dichloromethane (1 mL) was added $\mathrm{ZnBr}_{2}(25 \mathrm{mg}, 0.1 \mathrm{mmol})$ at $0{ }^{\circ} \mathrm{C}$. The mixture was stirred for $4 \mathrm{~h}$, quenched with saturated $\mathrm{NaHCO}_{3}$, and then extracted with dichloromethane. After the solvent was removed under reduced pressure, the residue was dissolved in dry DMF (0.5 mL). To this solution $N, N$-dimethyl-L-isoleucyl-L-leucine 13 (29 mg, 0.11 mmol), HATU (42 mg, $0.11 \mathrm{mmol}), \mathrm{K}_{2} \mathrm{CO}_{3}(30 \mathrm{mg}, 0.22 \mathrm{mmol})$ were added subsequently at 0 ${ }^{\circ} \mathrm{C}$. The mixture was stirred overnight, quenched with water, and then extracted with ethyl acetate. The combined organic layers were washed with water and brine, and dried over $\mathrm{Na}_{2} \mathrm{SO}_{4}$. The solution was concentrated and the residue was chromatographed to afford 1 (7 mg, $22 \%$ yield). ${ }^{1} \mathrm{H}$ NMR $\left(500 \mathrm{MHz}, \mathrm{CDCl}_{3}\right) \delta 0.86(\mathrm{~d}, J=$ $6.7 \mathrm{~Hz}, 3 \mathrm{H}), 0.94(\mathrm{~d}, J=6.9 \mathrm{~Hz}, 6 \mathrm{H}), 0.95(\mathrm{t}, J=6.8 \mathrm{~Hz}, 3 \mathrm{H}), 1.14(\mathrm{~m}, 1 \mathrm{H}), 1.48(\mathrm{~m}, 2 \mathrm{H}), 1.56(\mathrm{~m}$, 1H), $1.64(\mathrm{~m}, 1 \mathrm{H}), 1.75(\mathrm{~m}, 2 \mathrm{H}), 1.94(\mathrm{~m}, 2 \mathrm{H}), 2.24(\mathrm{~s}, 6 \mathrm{H}), 2.36(\mathrm{~m}, 1 \mathrm{H}), 2.46(\mathrm{~m}, 1 \mathrm{H}), 2.55(\mathrm{~d}, J$ $=5.6 \mathrm{~Hz}, 1 \mathrm{H}), 3.26(\mathrm{~m}, 1 \mathrm{H}), 3.57(\mathrm{~m}, 1 \mathrm{H}), 3.80(\mathrm{~s}, 3 \mathrm{H}), 4.21(\mathrm{~m}, 2 \mathrm{H}), 4.38(\mathrm{~d}, J=5.8 \mathrm{~Hz}, 1 \mathrm{H}), 4.52$ (dd, $J=9.0,4.2 \mathrm{~Hz}, 1 \mathrm{H}), 4.75(\mathrm{dd}, J=7.9,7.6 \mathrm{~Hz}, 1 \mathrm{H}), 5.94(\mathrm{~d}, J=8.8 \mathrm{~Hz}, 1 \mathrm{H}), 6.80$ (br s, $1 \mathrm{H})$, $6.82(\mathrm{~d}, J=7.3 \mathrm{~Hz}, 1 \mathrm{H}), 6.86(\mathrm{~d}, J=11.6 \mathrm{~Hz}, 1 \mathrm{H}), 6.92(\mathrm{dd}, J=11.5,8.8 \mathrm{~Hz}, 1 \mathrm{H}), 6.94(\mathrm{~d}, J=8.8$ $\mathrm{Hz}, 1 \mathrm{H}), 8.34(\mathrm{~d}, J=11.5 \mathrm{~Hz}, 1 \mathrm{H}) ;{ }^{13} \mathrm{C} \mathrm{NMR}\left(125 \mathrm{MHz}, \mathrm{CDCl}_{3}\right) \delta$ 11.7, 14.3, 21.4, 22.9, 24.4, 24.6, 
$26.7,28.8,32.4,34.0,40.6,42.8,45.1,47.6(2 \mathrm{C}), 55.7,61.8,62.5,74.1,78.4,106.4,110.5,113.6$, $116.7,121.4,123.9,150.7,151.0,167.5,171.0,171.3,171.6 ; \mathrm{MS} m / z 634.2[\mathrm{M}+\mathrm{Na}]^{+} ;$ HRMS Calcd. for $\mathrm{C}_{33} \mathrm{H}_{49} \mathrm{~N}_{5} \mathrm{O}_{6} \mathrm{Na}[\mathrm{M}+\mathrm{Na}]^{+}$634.3575, found 634.3578; IR (KBr) 802, $1025,1223,1260,1463,1509,1643,2852,2923,2958 \mathrm{~cm}^{-1} ;[\alpha]_{\mathrm{D}}{ }^{22}=-324.8(c=0.24$ in $\mathrm{CHCl}_{3}$ ). 


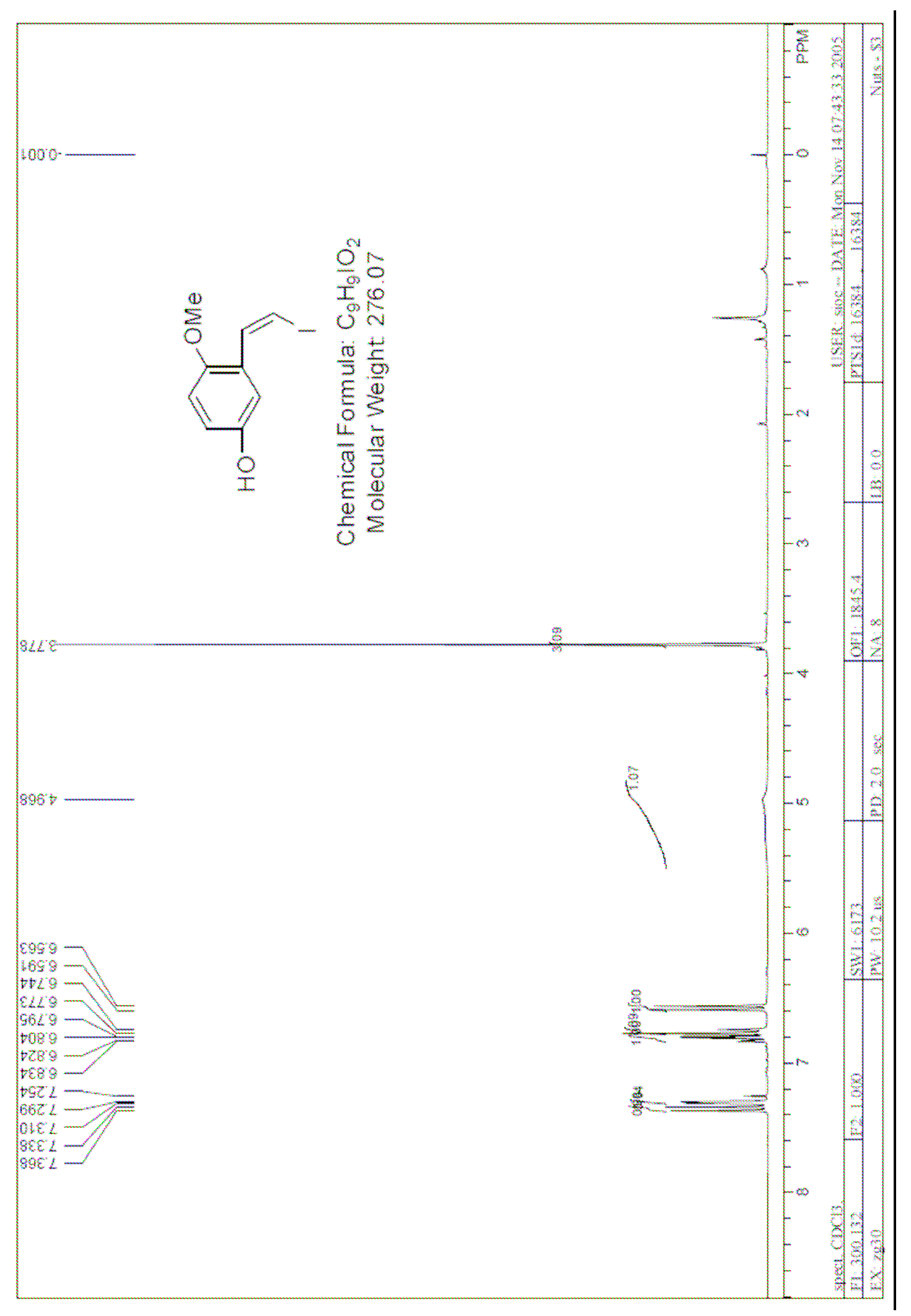




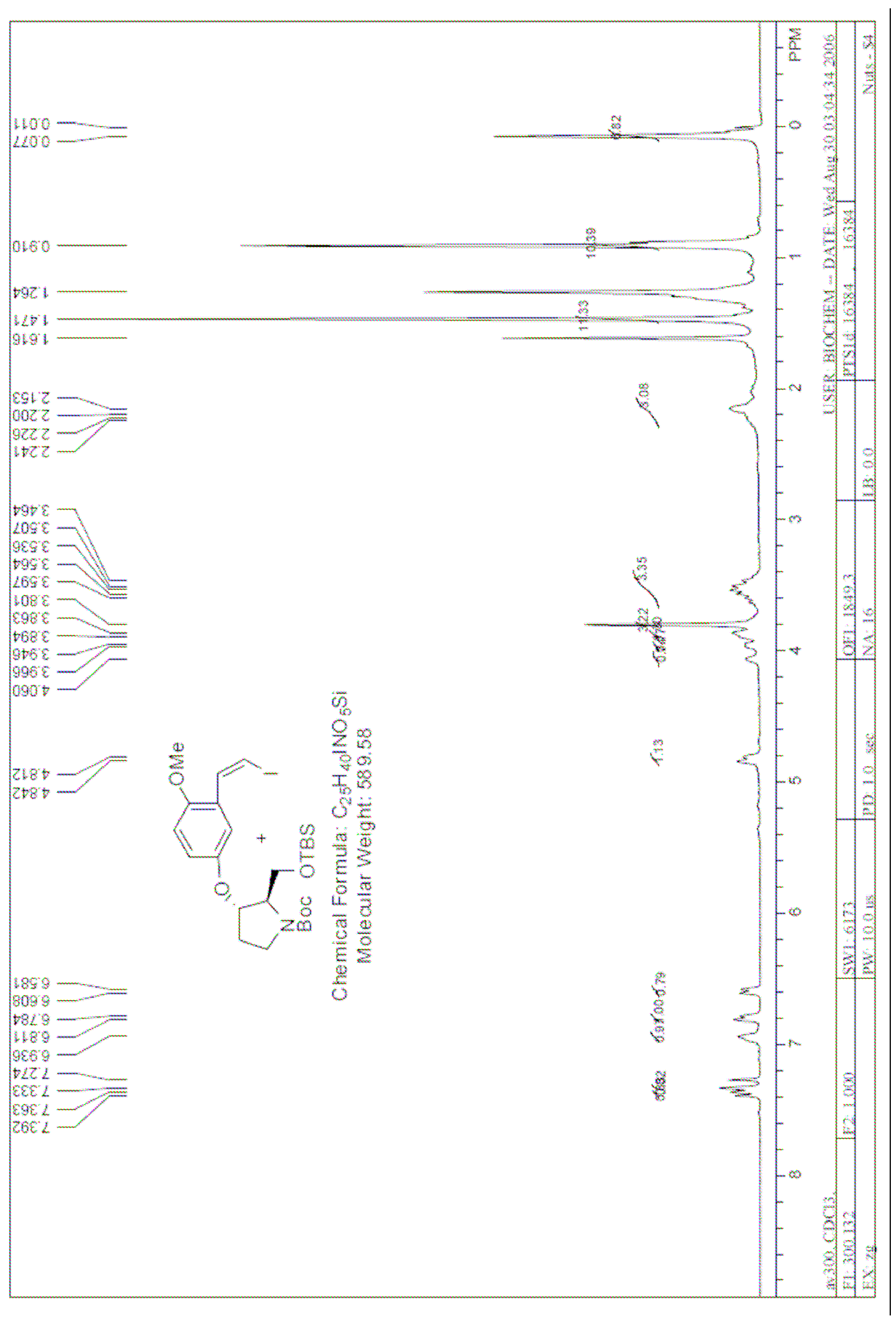




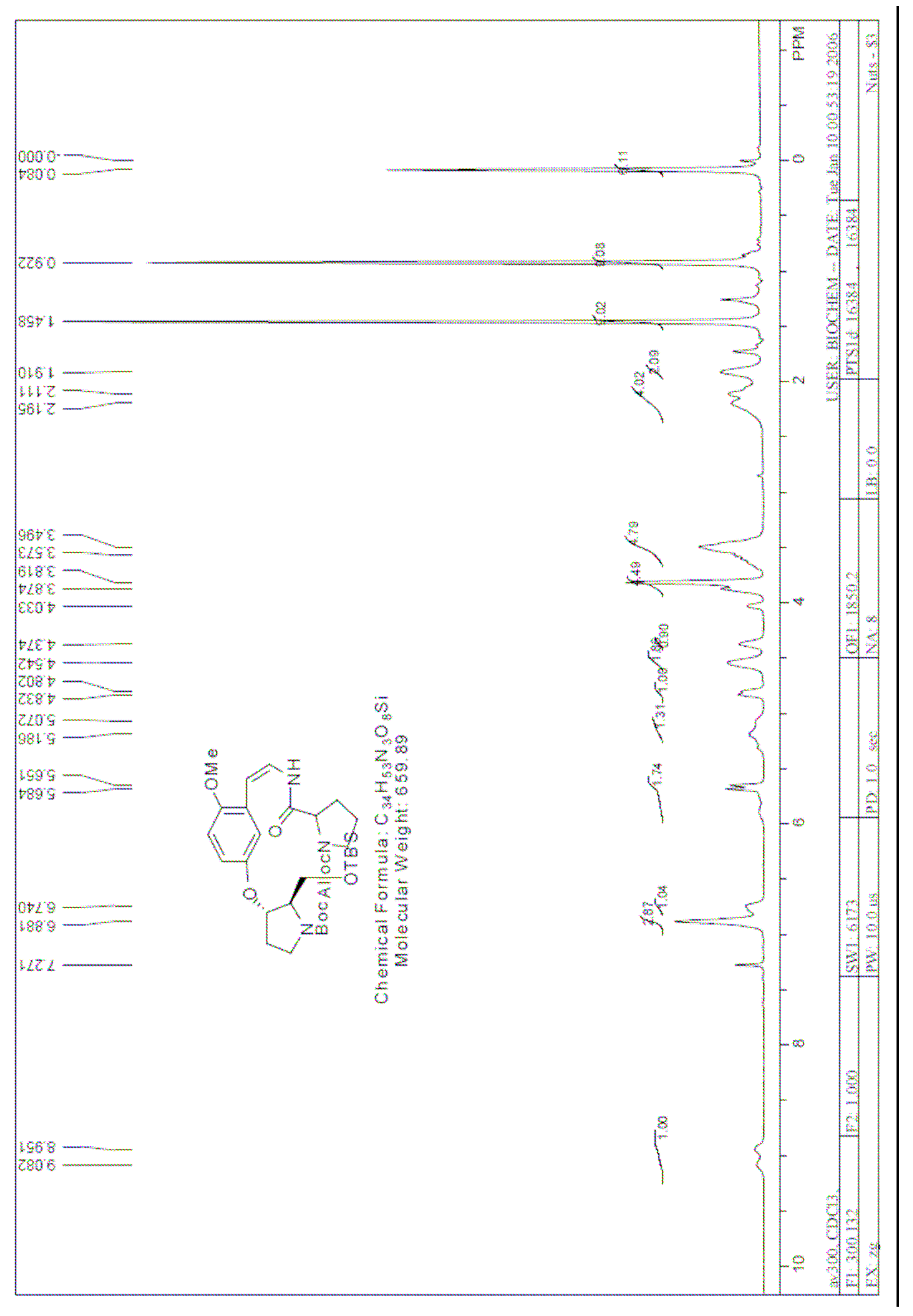




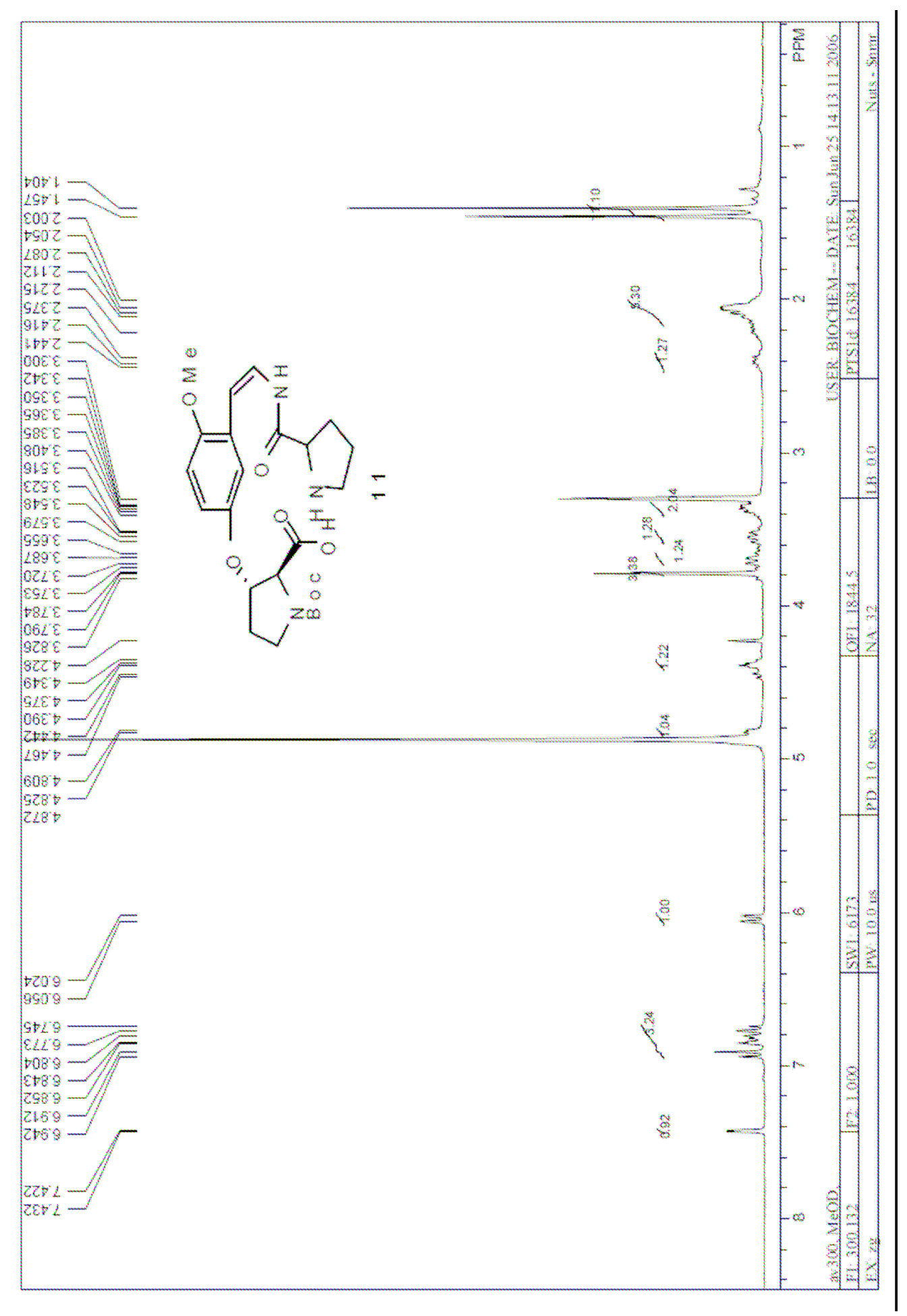




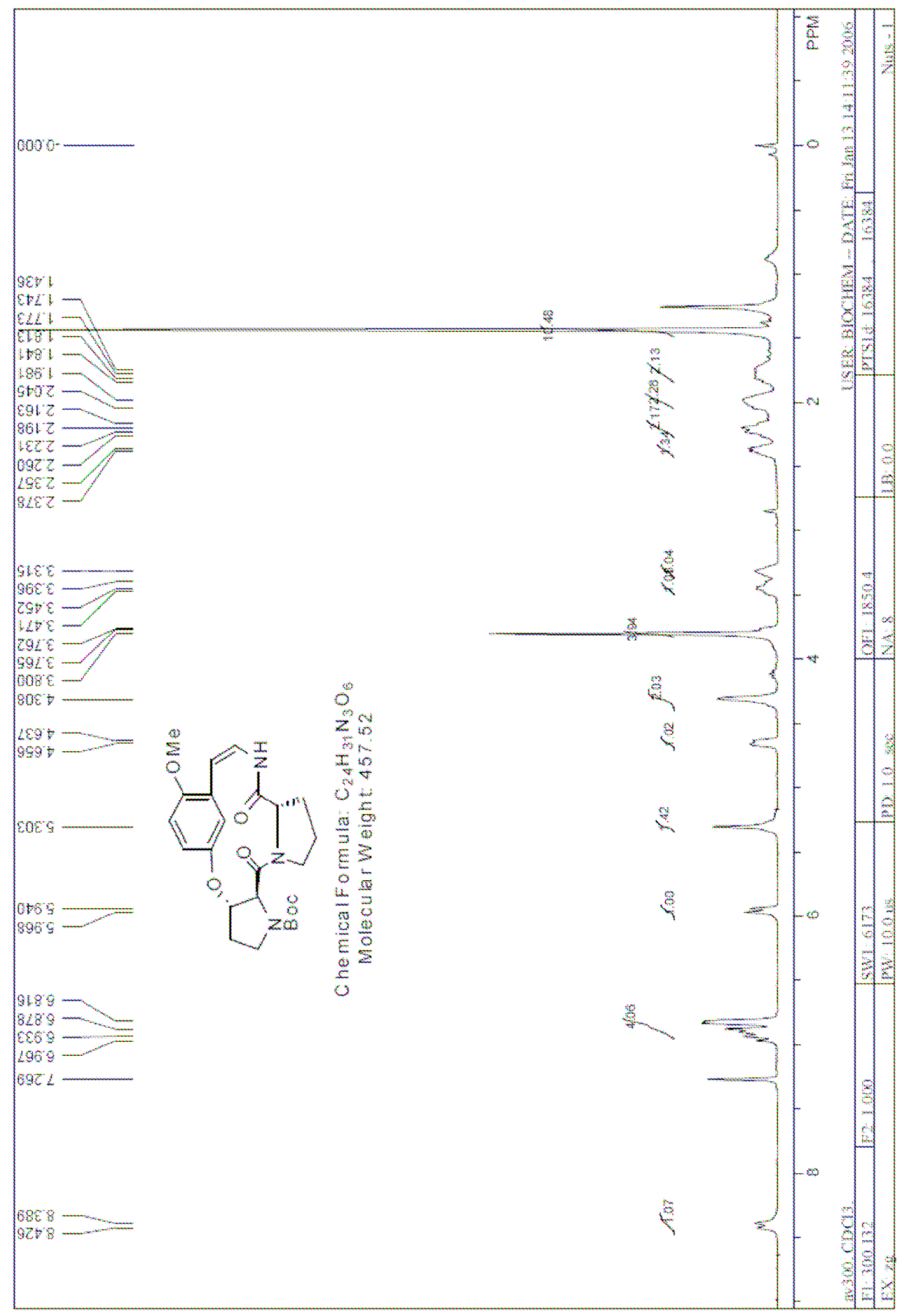




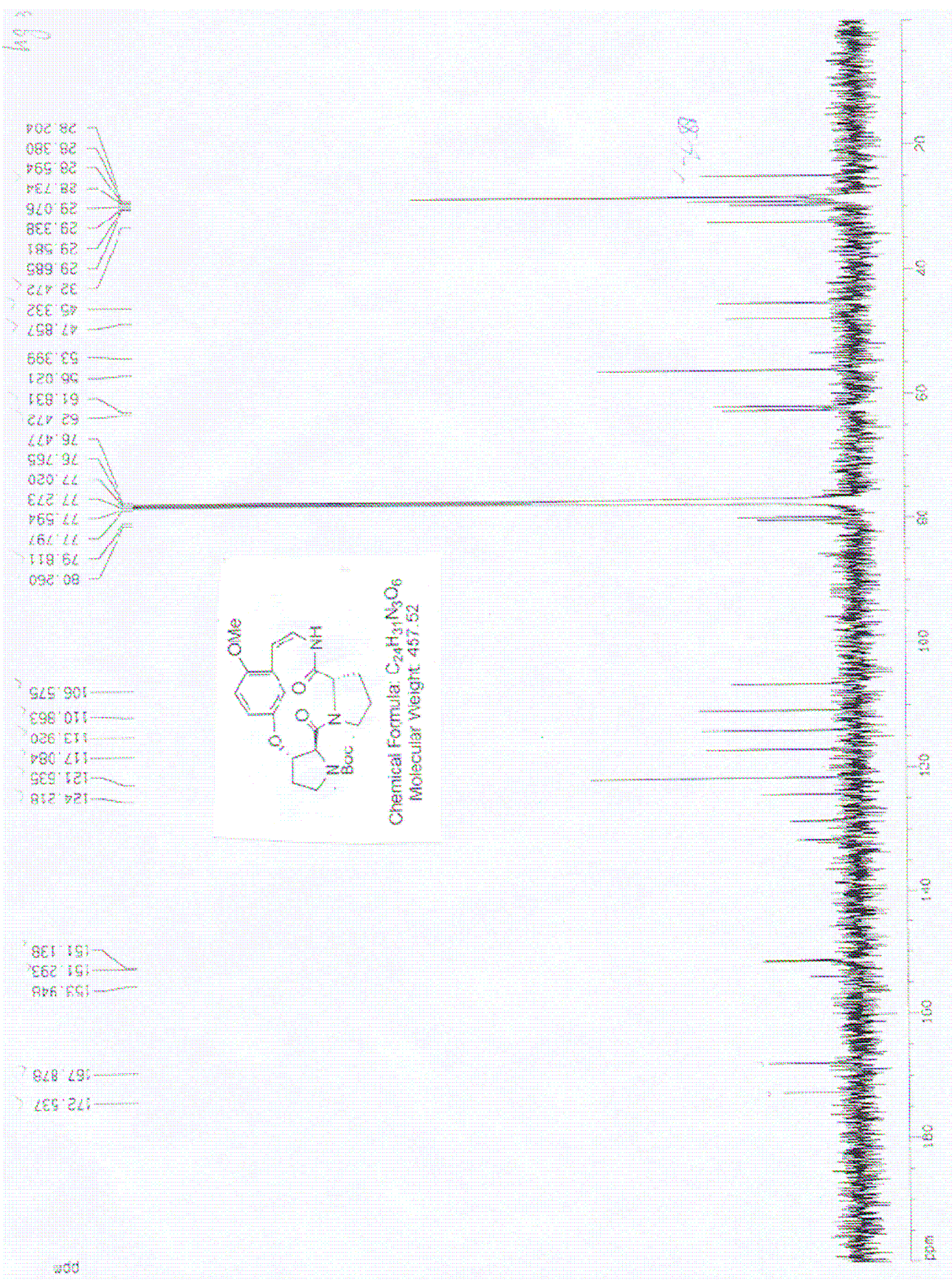




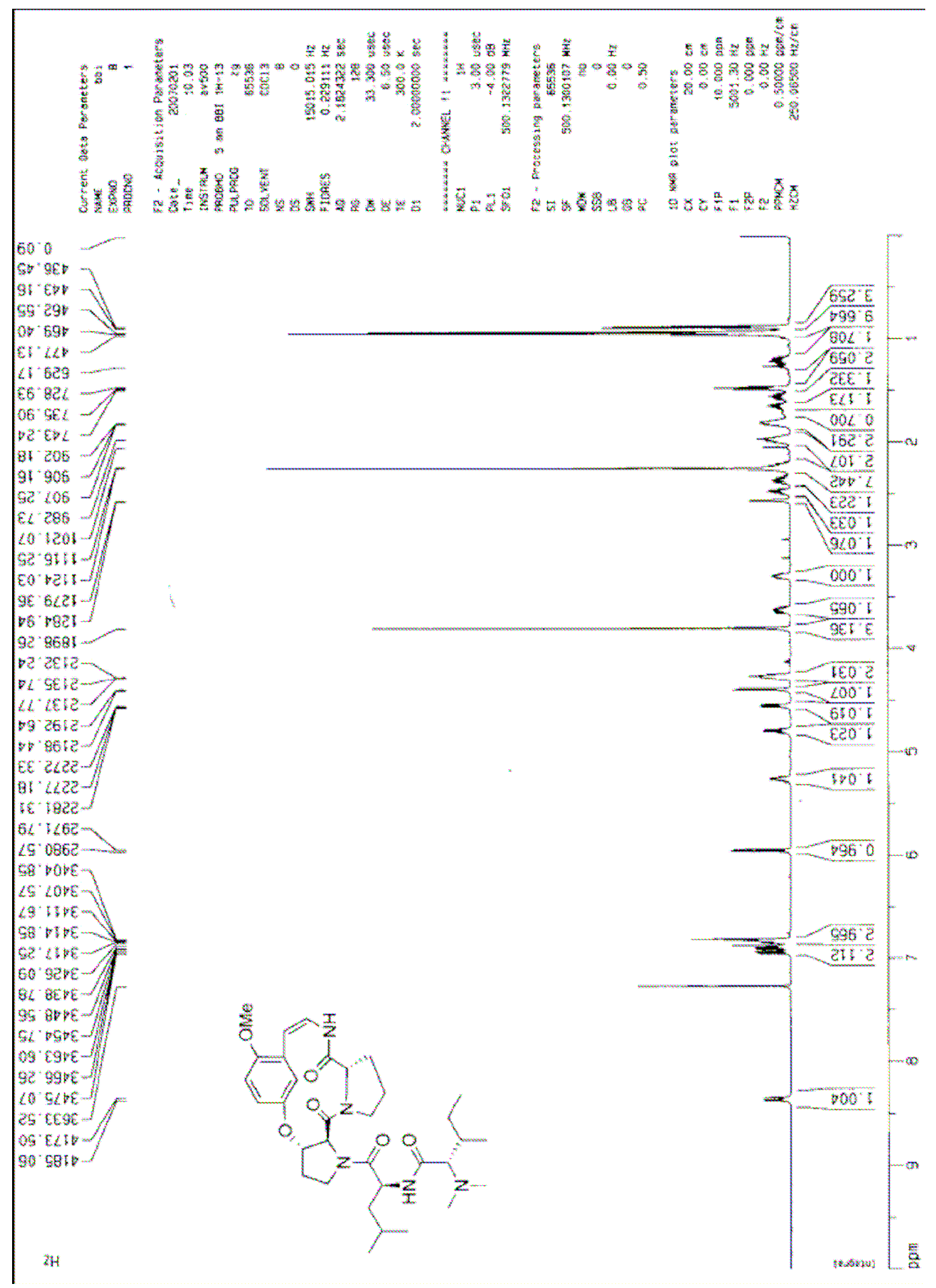


68911

CGE

940 t2

6zp $p ?$

91962

90< 92

$100+2$

tut 28

05075

Es? of - - -

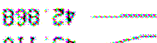

OIf

$\angle 19 \angle D$

ट26 99

a.t 15

26529

Eet?

5906

$52<96$

6959

ए6E 8 a.

E⿱t口: got -

ges or:

Orgel

एक 915

sto fel.......

TOSP E2t

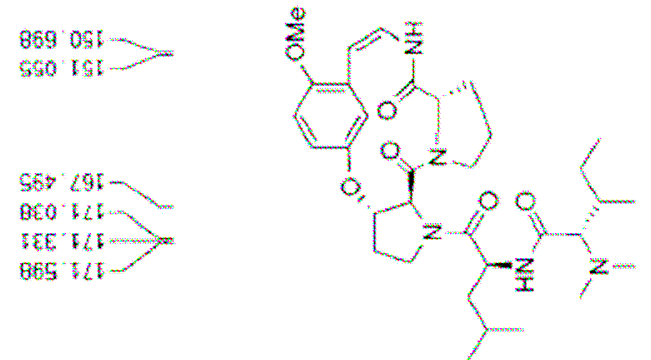

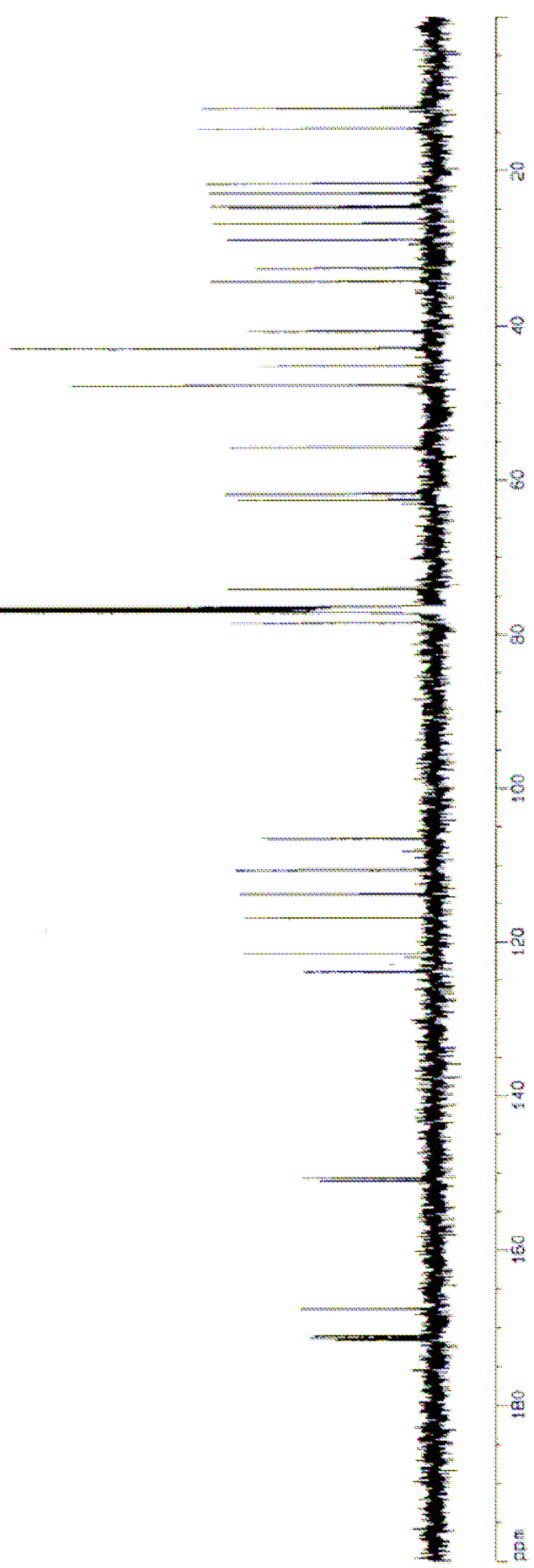

